\title{
Observation of Seeded Mn K $\beta$ Stimulated X-Ray Emission Using Two-Color X-Ray Free-Electron Laser Pulses
}

\author{
Thomas Kroll, ${ }^{1, *}$ Clemens Weninger $\odot,{ }^{2, \dagger}$ Franklin D. Fuller $\odot,{ }^{2}$ Marc W. Guetg $\odot,{ }^{3}$ Andrei Benediktovitch, ${ }^{4}$ Yu Zhang, ${ }^{5}$ \\ Agostino Marinelli, ${ }^{3}$ Roberto Alonso-Mori, ${ }^{2}$ Andy Aquila, ${ }^{2}$ Mengning Liang, ${ }^{2}$ Jason E. Koglin, ${ }^{2}$ Jake Koralek, ${ }^{2}$ \\ Dimosthenis Sokaras, ${ }^{1}$ Diling Zhu, ${ }^{2}$ Jan Kern $\odot,{ }^{6}$ Junko Yano, ${ }^{6}$ Vittal K. Yachandra, ${ }^{6}$ Nina Rohringer, ${ }^{4,7, \$}$ \\ Alberto Lutman $\oplus^{3}$ and Uwe Bergmann $\oplus^{5,8}$ \\ ${ }^{1}$ SSRL, SLAC National Accelerator Laboratory, Menlo Park, California 94025, USA \\ ${ }^{2}$ LCLS, SLAC National Accelerator Laboratory, Menlo Park, California 94025, USA \\ ${ }^{3}$ Accelerator Directorate, SLAC National Accelerator Laboratory, Menlo Park, California 94025, USA \\ ${ }^{4}$ Center for Free-Electron Laser Science, DESY, 22607 Hamburg, Germany \\ ${ }^{5}$ Stanford PULSE Institute, SLAC National Accelerator Laboratory, Menlo Park, California 94025, USA \\ ${ }^{6}$ Molecular Biophysics and Integrated Bioimaging Division, Lawrence, Berkeley National Laboratory, \\ Berkeley, California 94720, USA \\ ${ }^{7}$ Department of Physics, Universität Hamburg, 20355 Hamburg, Germany
}

(Received 3 December 2019; revised 25 May 2020; accepted 23 June 2020; published 17 July 2020)

\begin{abstract}
$\mathrm{K} \beta$ x-ray emission spectroscopy is a powerful probe for electronic structure analysis of $3 d$ transition metal systems and their ultrafast dynamics. Selectively enhancing specific spectral regions would increase this sensitivity and provide fundamentally new insights. Recently we reported the observation and analysis of $\mathrm{K} \alpha$ amplified spontaneous $\mathrm{x}$-ray emission from $\mathrm{Mn}$ solutions using an $\mathrm{x}$-ray free-electron laser to create the $1 s$ core-hole population inversion [Kroll et al., Phys. Rev. Lett. 120, 133203 (2018)]. To apply this new approach to the chemically more sensitive but much weaker $\mathrm{K} \beta$ x-ray emission lines requires a mechanism to outcompete the dominant amplification of the $\mathrm{K} \alpha$ emission. Here we report the observation of seeded amplified $\mathrm{K} \beta$ x-ray emission from a $\mathrm{NaMnO}_{4}$ solution using two colors of $\mathrm{x}$-ray free-electron laser pulses, one to create the $1 \mathrm{~s}$ core-hole population inversion and the other to seed the amplified $\mathrm{K} \beta$ emission. Comparing the observed seeded amplified $\mathrm{K} \beta$ emission signal with that from conventional $\mathrm{K} \beta$ emission into the same solid angle, we obtain a signal enhancement of more than $10^{5}$. Our findings are the first important step of enhancing and controlling the emission of selected final states of the $\mathrm{K} \beta$ spectrum with applications in chemical and materials science.
\end{abstract}

DOI: 10.1103/PhysRevLett.125.037404

With its sensitivity to spin state, oxidation, and ligand environment, $\mathrm{K} \beta$ x-ray emission spectroscopy (XES) has long been employed to gain electronic structure information of $3 d$ transition metal complexes [1-7]. Unlike x-ray absorption spectroscopy, $\mathrm{K} \beta$ XES is predominantly sensitive to the number of unpaired $3 d$ electrons and less to the local geometry $[6,8]$, and it has a higher chemical sensitivity than $\mathrm{K} \alpha$ XES [4]. XES does not require a monochromatic X-ray beam and can be applied simultaneously with structural probes, such as X-ray diffraction and scattering [9-10]. Besides its weak signal, the main limitation of $\mathrm{K} \beta \mathrm{XES}$ is the core-hole lifetime broadening that washes out the subtle spectral response to electronic structure changes. In this Letter, we report the creation of strongly enhanced seeded $\mathrm{K} \beta$ stimulated $\mathrm{x}$-ray emission in a manganese solution. This nonlinear method has the potential for amplifying individual spectral features characteristic of the electronic structure and suppressing the core-hole lifetime broadening. With future optimized experimental conditions, this enhanced spectral sensitivity and control will widen the application of $\mathrm{K} \beta$ XES.
Nonlinear x-ray spectroscopy [11-17] requires the high peak power of an X-ray free-electron laser source [18-24]. An important case is amplified-spontaneous emission (ASE) or single-pass atomic x-ray lasing. Here, an intense $\mathrm{x}$-ray pump pulse is tuned above an inner-shell absorption edge energy, creating a population inversion along its pathway through an optically thick medium (several absorption lengths). Spontaneously emitted photons along the direction of the population inversion path can stimulate the emission at the same wavelength and direction, creating ASE. When the collective emission time is short compared to the decoherence time, ASE can also be referred to as superfluorescence $[25,26]$. We have recently measured the $\mathrm{K} \alpha$ ASE spectra from two different manganese solutions to study how stimulated $\mathrm{x}$-ray emission spectroscopy (S-XES) can be applied to $3 d$ transition metal ions [14]. We found that ASE exhibits a chemical shift that is characteristic of the manganese ion and observed strong gain narrowing with spectral widths far below the $1 s$ core-hole lifetime broadening. For the weaker $\mathrm{K} \beta$ lines, $\mathrm{K} \beta$ ASE can only 


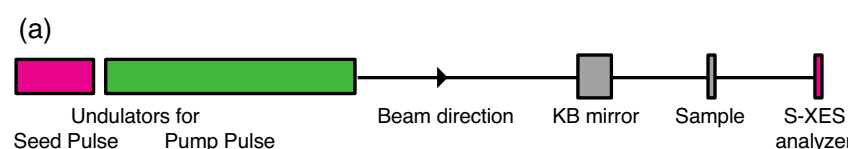

(b)

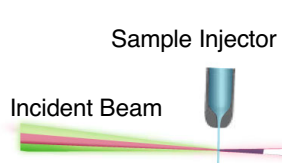

(c)

valence levels
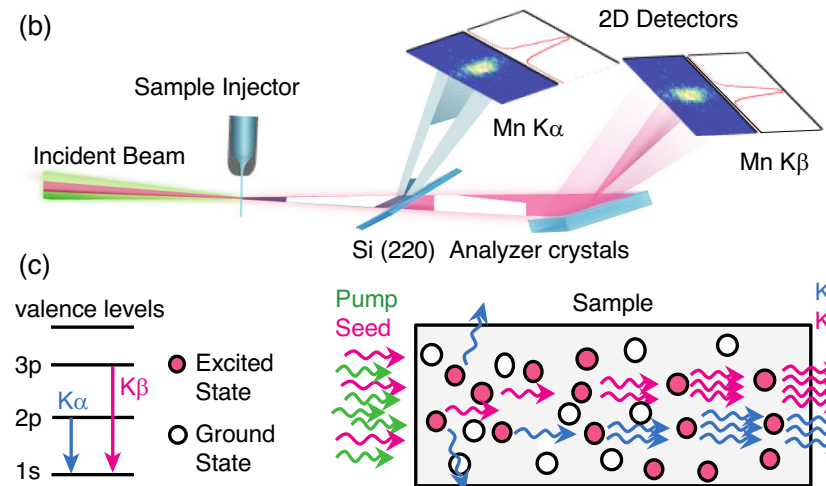

Si (220) Analyzer crystals

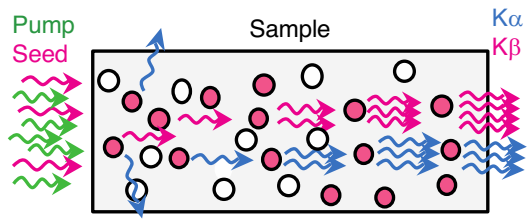

FIG. 1. (a): Schematics of the beam line layout. (b) Experimental setup using a partially transparent $\mathrm{K} \alpha$ analyzer and $\mathrm{K} \beta$ analyzer for simultaneous collection of $\mathrm{K} \alpha$ ASE and seeded $\mathrm{K} \beta$ S-XES signals. (c) Schematics of the stimulated emission processes.

occur once the $\mathrm{K} \alpha$ amplification is saturated, requiring unrealistically high peak power [14]. Seeding the stimulated emission of $\mathrm{K} \beta$ overcomes this limitation. Here, the pump pulse is tuned above the absorption edge to create the population inversion, and the seed pulse is tuned to the $\mathrm{K} \beta$ energy. The equivalent mechanism was initially used to seed the $\mathrm{K} \alpha$ line in a $\mathrm{Cu}$ metal foil [13]. Seeding the weaker $\mathrm{K} \beta$ line in a solution is much more challenging and requires sufficient spatiotemporal overlap of pump and seed pulse to outcompete the much stronger $\mathrm{K} \alpha$ ASE.

Experiments were performed at the Coherent X-ray Imaging (CXI) instrument [27] at the Linac Coherent Light Source. We used the split undulator method [28] with strong pulse compression [29] to create the required pump/seed power and overlap for the two colors [Fig. 1(a)]. The first seven undulators created the $\sim 4 \mu \mathrm{J}$ seed pulse, while the last 23 undulators created the $\sim 500 \mu \mathrm{J}$ pump pulse. Both pulses were focused to $\sim 150 \mathrm{~nm}$ diameter at the sample position using $\mathrm{KB}$ mirrors with an estimated focal depth of $100 \mu \mathrm{m}[30,31]$. A $200 \mu \mathrm{m}$ diameter liquid jet was used for sample delivery of 4 molar $\mathrm{NaMnO}_{4}$ solution obtained from Sigma-Aldrich. For the $\mathrm{K} \alpha$ and $\mathrm{K} \beta$ emission analysis, we used two flat $\mathrm{Si}$ (220) analyzer crystals at respective Bragg angles of $33.18^{\circ}$ and $29.80^{\circ}$ diffracting the signal onto $2 \mathrm{D}$ detectors [32]. The two crystals were mounted serially, allowing the simultaneous analysis of both emission lines [Fig. 1(b)]. The first crystal, used for $\mathrm{K} \alpha$ analysis, was semitransparent with a thickness of $10 \mu \mathrm{m}$, absorbing $38 \%$ of the photons in the $\mathrm{K} \beta$ energy region at $6.49 \mathrm{keV}$, while fully reflecting the $\mathrm{K} \alpha$ photons at $5.9 \mathrm{keV}(\sim 3.7 \mu \mathrm{m}$ extinction length along the incident $\mathrm{x}$-ray path). The second crystal, used for $\mathrm{K} \beta$ analysis, was thick with a $1^{\circ}$ asymmetrical cut [14]. The $\sim 2 \mathrm{mrad}$ vertical
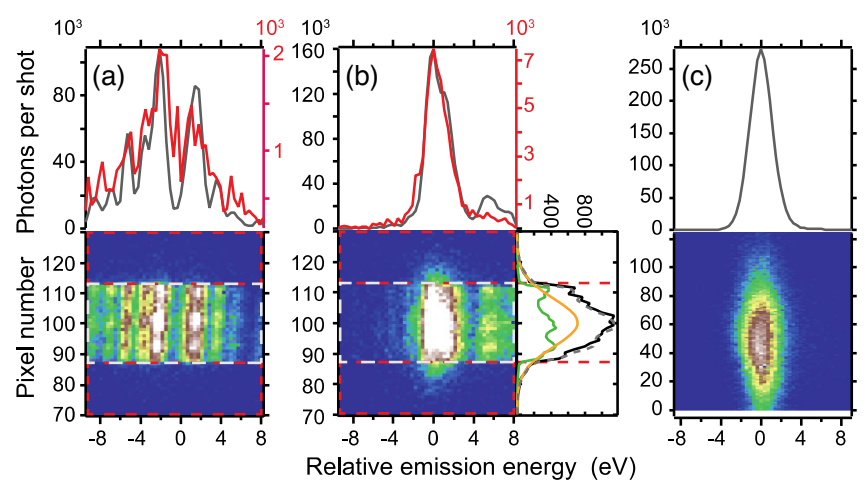

FIG. 2. 2D single shot images and spectra in the $\mathrm{K} \beta$ energy region taken without sample (a) and with sample (b). The dark gray curves show the spectra integrated over whole spatial range, while for the red curves only the range above and below the SASE box was used. $0 \mathrm{eV}$ relative energy corresponds to the maximum of the $\mathrm{K} \beta$ XES spectrum $(6489.3 \mathrm{eV})$. The plots on the right of (b) show spatial distributions integrated over 5 pixels $(1.8 \mathrm{eV})$ around the maximum at $0 \mathrm{eV}$. It shows the full distribution (black), a Gaussian fit to the region outside the SASE box representing the seeded S-XES signal (orange), the corresponding SASE contribution (green) that when added to the Gaussian (gray dashed) reproduces the full distribution (black). Single-shot K $\alpha$ ASE image and spectrum at $5898.1 \mathrm{eV}$ (c).

divergence of the $\mathrm{x}$-ray beam emerging from the focus results in an energy range of $\sim 20 \mathrm{eV}$ for each analyzer. The energy of the pump pulse was set above the Mn K edge to $6.6 \mathrm{keV}$, while the seed pulse energy was set to the $\mathrm{Mn} \mathrm{K} \beta$ line at $6.49 \mathrm{keV}$. Both pulses had a pulse length of $\sim 10 \mathrm{fs}$ using the SASE process without further monochromatization $\left(\Delta E / E \sim 5 \times 10^{-3}\right)$. The $\mathrm{K} \alpha$ and $\mathrm{K} \beta$ energy axes on the $2 \mathrm{D}$ detectors were calibrated using the transmission through thin diamond crystal in the upstream section of the CXI instrument. This gives an accurate relative energy calibration for each detector pixel and allows the absolute energy calibration to within a few $\mathrm{eV}$. For calibration, we used the maxima of synchrotron spectra of $\mathrm{NaMnO}_{4}$ at $5898.1 \mathrm{eV}$ for $\mathrm{K} \alpha$ and $6489.3 \mathrm{eV}$ for $\mathrm{K} \beta$ measured at SSRL [33]. The relative energies in Figs. 2 and 4 are displayed with respect to these values. Figure 1(c) shows the energy level diagram of the $\mathrm{K} \alpha$ and $\mathrm{K} \beta$ XES processes following the excitation of the $1 s$ electron into the continuum. The $\mathrm{Mn} \mathrm{K} \alpha$ emission at $\sim 5.9 \mathrm{keV}$ is $\sim 7$ times stronger than the $\mathrm{K} \beta$ emission at $\sim 6.49 \mathrm{keV}$ [34]. To ensure the highest cross section for creating population inversion [11,15,35], we set the pump pulse just above the $1 s$ ionization energy. In ASE the transition with the largest dipole transition moment, i.e., $\mathrm{K} \alpha_{1}$, gets amplified first and weaker lines only get amplified once the $\mathrm{K} \alpha_{1}$ emission is saturated. To stimulate the amplification of the weaker $\mathrm{K} \beta$ transition, a seed pulse is tuned to its respective energy. Such seeding can at least partially outcompete the K $\alpha$ ASE. The concept of K $\alpha$ ASE and seeded $\mathrm{K} \beta$ S-XES is shown in Fig. 1(c) (right). The excited Mn ions along the beam path (solid red circles) 
decay spontaneously, emitting predominantly $\mathrm{K} \alpha$ photons, with those in the direction of the population inversion initiating the $\mathrm{K} \alpha \mathrm{ASE}$ process (blue arrows). In some parts of the sample, the seed pulse (red) outcompetes the $\mathrm{K} \alpha$ ASE, leading to seeded $\mathrm{K} \beta$ S-XES. The seed pulse energy needs to match a $K \beta$ transition energy and overlap with the pump pulse both spatially and temporally within the $1 \mathrm{~s}$ core-hole lifetime $(\sim 1 \mathrm{fs})$. This is challenging given the small pump and seed pulse sizes, the small Mn absorption cross section, and the short $1 s$ core-hole lifetime. When using a SASE seed pulse, only a very small percentage of the photons contribute to creating the seeded $\mathrm{K} \beta \mathrm{S}$-XES, and the process occurs simultaneously with $\mathrm{K} \alpha$ ASE.

The $\mathrm{K} \beta$ analyzer selects photons in the energy range of the $\mathrm{K} \beta$ line and the SASE seed pulse (Figure 1). Without an upstream spectrometer that measures the seed pulse at every shot, it is challenging to separate it from the seeded $\mathrm{K} \beta$ S-XES. Fortunately, the seeded $\mathrm{K} \beta \mathrm{S}$-XES has a larger angular divergence than the seed pulse. Figure 2(a) shows an example of a measured single-shot spectrum of the incoming seed pulse without the sample. The horizontal and vertical axes reflect the energy dispersion and beam divergence, respectively. In the nondispersive direction (vertical in Fig. 2), the beam extends over 25 pixels $(2.75 \mathrm{~mm})$ and is sharply cut by overfilling the upstream beam line KB mirror optics. Figure 2(b) shows a single shot spectrum including the sample. The area corresponding to the seed pulse [indicated as a light gray dashed rectangle in 2(a) and 2(b)] contains both the SASE seed pulse plus the seeded $\mathrm{K} \beta$ S-XES signals. In the maximum intensity region, we observe an additional signal extending over the sharp edges of the seed pulse region at $0 \mathrm{eV}$ relative energy. This signal originates from seeded $\mathrm{K} \beta$ S-XES. Similar observations of excess signal divergence were made previously for experiments in neon $[11,15]$. A typical K $\alpha$ ASE shot is shown in Fig. 2(c). Note that both emission signals of $\mathrm{K} \alpha$ and $\mathrm{K} \beta$ exhibit a larger divergence than the transmitted pump pulse. The seeded $\mathrm{K} \beta$ S-XES divergence at $0 \mathrm{eV}$ relative energy extends over 40 pixels $(4.4 \mathrm{~mm}=1.3 \mathrm{mrad})$, while the stronger $\mathrm{K} \alpha$ divergence extends over more than 100 pixels $(11 \mathrm{~mm}=3.1 \mathrm{mrad})$. The difference between the $\mathrm{K} \alpha$ ASE and seeded $\mathrm{K} \beta$ S-XES beam divergence can be understood as follows: In K $\alpha$ ASE, the amplification process is spontaneous and can occur in every direction with sufficiently large population inversion. As the gain length is shorter than the focal depth of the pump pulse, the dispersion of the ASE beam can be much larger than that of the pump pulse. The origin of the seeded $\mathrm{K} \beta$ S-XES divergence is different. $\mathrm{K} \beta$ photons are amplified by a seed photon with the same energy, direction, and phase. Therefore, in principle, the seeded $\mathrm{K} \beta \mathrm{S}$-XES divergence matches that of the seed pulse. The experimentally observed larger divergence can be explained by the fact that any small photon source has a minimal divergence given by the transform limit, which is

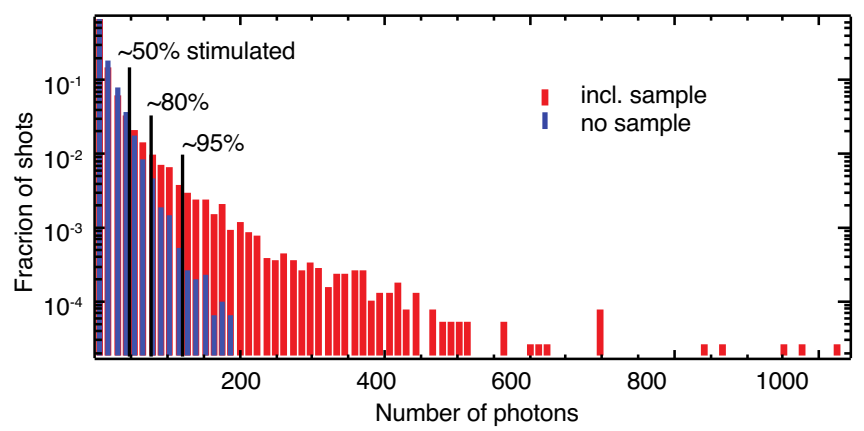

FIG. 3. Histograms of the intensity outside the SASE box within a 5-pixel $(1.8 \mathrm{eV})$ region around the maximum with the sample (red) and without the sample (blue). The three vertical lines represent example thresholds for seeded $\mathrm{K} \beta$ S-XES probabilities of $\sim 50 \%, \sim 80 \%$, and $\sim 95 \%$.

$\Delta \theta=(\lambda / a)$, where $\lambda$ is the wavelength and $a$ is the size of the source (see the Fraunhofer diffraction as an analog [36]). For a fully coherent beam the transform limit is $\Delta \theta_{c}=(\lambda / 4 \pi a)$ [37]. In our observed seeded $\mathrm{K} \beta$ S-XES, $\Delta \theta$ is determined by the ratio of the signal width on the detector $[\sim 18$ pixels $=2 \mathrm{~mm}$ FWHM, Fig. 2(b) $]$ and the sample-detector distance $(3.5 \mathrm{~m})$ resulting in $\Delta \theta=$ $(2 \mathrm{~mm} / 3.5 \mathrm{~m})=0.57 \times 10^{-3}$. Assuming that the seeded $\mathrm{K} \beta \mathrm{S}$-XES signal originates from a fully coherent source, this corresponds to an estimated source size of $a \sim 27.3 \mathrm{~nm}$. Our observed divergence thus indicates that the effective source size leading to seeded $\mathrm{K} \beta \mathrm{S}$-XES is significantly smaller than the $\sim 150 \mathrm{~nm}$ for the pump and seed pulses. This is not unexpected as only a small fraction of the seed pulse has spatiotemporal overlap with the pump pulse.

We use the signals that extend to outside the seed pulse region [red dashed rectangles in Figs. 2(a) and 2(b)] to distinguish between the seeded $\mathrm{K} \beta$ S-XES and the seed pulse. Figure 2 (top) shows the integrated spectra of the signals. The gray curves are the integrated intensity over the entire spatial direction (i.e., light gray plus two red dashed boxes), while the red lines originate from the signal outside the seed pulse (two red dashed boxes). Integrating over the entire spatial direction that originates from both seeded $\mathrm{K} \beta$ S-XES and SASE seed pulse (gray curve) shows two spectral features [Fig. 2(b)]. When excluding the signal inside the seed pulse box, we observe only one peak (red curve).

We now analyze the difference between seed pulse and seeded K $\beta$ S-XES in more detail. Figure 3 shows the log scale histograms of shots with varying the intensities outside the seed pulse box in the energy region of \pm 2 pixels around the maximum intensity measured with and without sample. To account for the different number of shots (37 999 with sample and 30324 without sample) we normalized them to 1 . Intensities in the dataset without sample are multiplied by 0.65 to account for beam attenuation. Both datasets give a similar distribution for shots with small intensities (no seeded $\mathrm{K} \beta$ S-XES signal), 


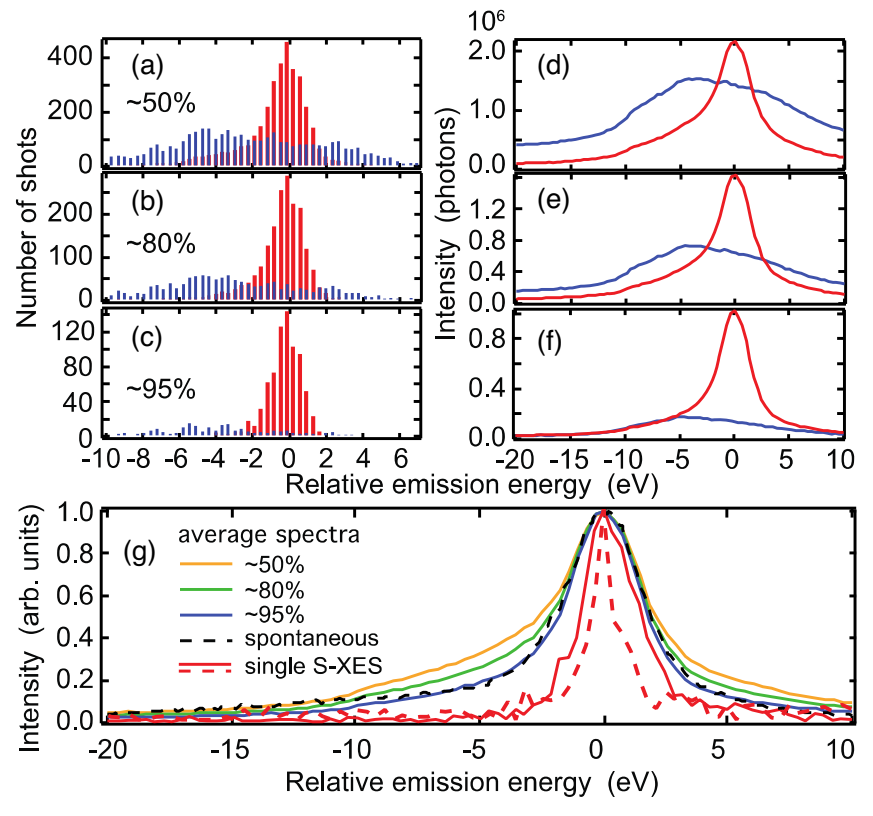

FIG. 4. Histograms of the maximum peak position (a)-(c) and average spectra (d)-(f) for the seed SASE only (blue) and the seeded K $\beta$ S-XES signals (red). The three thresholds (Fig. 3) are used for (a) and (d) ( $\sim 50 \%$ chance of seeded K $\beta$ S-XES), (b) and (e) $(\sim 80 \%)$, and (c) and (f) $(\sim 95 \%)$. The three average seeded $\mathrm{K} \beta$ $\mathrm{S}$-XES spectra (orange, green, blue), the single-shot spectrum from Fig. 2(b) (red solid line) and a very narrow shot (red dashed line) are normalized to one and compared to the $\mathrm{NaMnO}_{4}$ spontaneous XES spectrum taken at a synchrotron (black dashed line). Energies are relative to the energy of the peak maximum.

while only the dataset with the sample shows shots with higher intensities. We define three thresholds: At threshold 1 , where the two histograms start to diverge, the probability $p=\left[\left(I_{\text {sample }}-I_{\text {no sample }}\right) / I_{\text {sample }}\right]$ to detect seeded $\mathrm{K} \beta$ S-XES photons above this threshold 1 is $\sim 50 \%$, above threshold 2 it is $\sim 80 \%$, and above threshold 3 it is $\sim 95 \%$. Figures 4(a)-4(c) show the histograms of the position of the peak maximum for all shots above the three thresholds with sample (red bars) and without (blue). While the nosample histograms show a random distribution of the peak maxima, the with-sample histograms exhibit a sharp distribution. Comparing the averaged spectra for the three thresholds [Figs. 4(d)-4(e)] also shows a stark difference. The no-sample spectra reflect the typical averaged SASE energy distribution whereas the spectra with sample show a single sharp peak indicative of an increasing seeded $\mathrm{K} \beta$ S-XES contribution. Comparison of the three averaged spectra [Fig. 4(g), orange, green, blue] shows a narrowing and reduced intensity in the wings with increasing probability of seeded $\mathrm{K} \beta$ S-XES and decreasing contribution of the SASE pulse. The $295 \%$ spectrum (blue) shows a similar width in the main line and a lower intensity in the tails compared to spontaneous XES (black dashed line). This difference is not surprising because the stronger features in the $\mathrm{K} \beta$ main region get stimulated more easily.
The fact that the average of seeded $\mathrm{K} \beta$ S-XES spectra can reproduce the spontaneous XES spectrum indicates the chemical sensitivity of our approach. Comparing the single-shot seeded $\mathrm{K} \beta$ S-XES spectrum from Fig. 2(b) (red solid line) with spontaneous XES shows spectral narrowing as previously observed in $\mathrm{K} \alpha \mathrm{ASE}$. While this is one of the strongest shots, we also found weaker shots with widths below $1.5 \mathrm{eV}$ FWHM. An example is shown in Fig. 2 (red dashed line). As these single-shot spectra have small contributions from the SASE seed pulse, there remains some uncertainty about their exact spectral shapes. Better seed pulse diagnostics and monochromatic seed pulses (see discussion in Ref. [38]), will provide even significantly narrower single shot seeded $\mathrm{K} \beta$ S-XES spectra. This will allow us to further sharpen and control the spectral shape by tuning the seed pulse to a specific resonance and operating in the gain-narrowing regime.

To estimate the signal enhancement of seeded K $\beta$ S-XES we compare the number of stimulated emission photons with the number of spontaneous emission photons into the same solid angle. While the spontaneous $\mathrm{K} \beta$ emission was too weak to detect with our setup, we get a very good estimate from the previously measured spontaneous $\mathrm{K} \alpha$ emission performed in the same experimental geometry, where we detected approximately one $\mathrm{K} \alpha$ photon per $2.5 \mathrm{~mJ}$ pulse [14]. When correcting for the $0.5 \mathrm{~mJ}$ pulse energy and seven times lower $\mathrm{K} \beta$ yield in the current experiment, this translates to $1 /[(2.5 / 0.5) * 7] \sim 0.029$ spontaneous $\mathrm{K} \beta$ photons per pulse. To estimate the number of seeded K $\beta$ S-XES photons per shot, we fitted a Gaussian line shape to the tails of the spatial distribution that falls outside the SASE box [i.e., outside the gray dashed rectangular, orange curve in Fig. 2(b)], while ensuring that the total signal inside the SASE box (K $\beta$ S-XES plus SASE seed pulse) fits the observed signal [black curve in Fig. 2(b)]. We estimated the SASE seed pulse signal (green curve) by approximating it with the neighboring intensity at $\sim 5.5 \mathrm{eV}$ (where there is no $\mathrm{K} \beta \mathrm{S}$-XES signal) and varying its intensity until it fits the experimental spatial distribution (gray dashed curve). The integral under the Gaussian (orange line) estimates the number of $\mathrm{K} \beta \mathrm{S}$-XES photons per pulse to $\sim 14000$. Even considering a large error in this estimate due to the uncertainly of the Gaussian interpolation, the signal enhancement we obtain $(\sim 14000 / 0.029)$ is larger than $10^{5}$.

Using two analyzer crystals [Fig. 1(b)] allows the simultaneous detection of $\mathrm{K} \alpha$ ASE and seeded $\mathrm{K} \beta$ S-XES signals originating from the same pump pulse. Figure 5 shows the correlation plots of $\mathrm{K} \beta$ vs $\mathrm{K} \alpha$ emission intensity plotted on a double log scale. For the seeded K $\beta$ S-XES data, only the intensity outside the seed pulse region that has an intensity above the $\sim 95 \%$ threshold (Fig. 3) was used. We observe a large distribution of $\mathrm{K} \alpha$ ASE and seeded $\mathrm{K} \beta$ S-XES intensities. Taking only the maximum seeded $\mathrm{K} \beta$ S-XES intensity points for each $\mathrm{K} \alpha$ intensity results in an almost linear increase on the double logarithmic scale. The better 


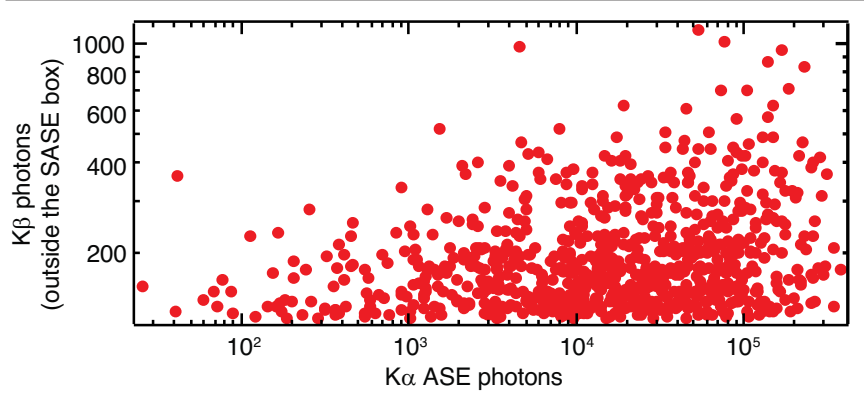

FIG. 5. Correlation plots of seeded $\mathrm{K} \beta$ S-XES vs $\mathrm{K} \alpha$ ASE intensities plotted on a double log scale. All K $\beta$ intensities outside the seed SASE box representing seeded $\mathrm{K} \beta \mathrm{S}$-XES signals with a $\sim 95 \%$ probability are shown.

the population inversion, the stronger is the K $\alpha$ ASE signal and the better is the chance for creating a strong seeded $\mathrm{K} \beta$ $\mathrm{S}$-XES signal. Figure 5 shows that in our experiment seeded $\mathrm{K} \beta$ S-XES did not yet reach saturation, but stayed in the linear gain regime. The stochastic nature of the SASE seed pulse and the tight requirements for spectral, temporal and spatial overlap lead to a large variation in seeded $\mathrm{K} \beta$ S-XES signals even for identical population inversions.

Much improved spectral and temporal pulse diagnostic and control $[39,40]$ will remove many of these uncertainties in the future. Shot-by-shot diagnostics of the seed pulse will make it possible to separate the seeded $\mathrm{K} \beta$ S-XES signal from the seed pulse. Using a monochromatic seed pulse will enable the stimulation of selected final states, and using a monochromatic pump pulse will enable the population inversion of selected excited states. This will make it possible to separate and control various spectral contributions to the $\mathrm{K} \beta$ line such as final state energies and core-hole lifetime broadening through gain narrowing. Such enhanced sensitivity to the electronic structure of $3 d$ transition metal compounds will enable a wide range of applications, including complex systems and studies of ultrafast intersystem crossings in light-harvesting and photocatalytic molecules. While prototypical model compounds can be characterized with spontaneous $\mathrm{K} \beta$ XES [6], natural systems and those employed for practical solar energy conversion may exhibit much more subtle changes that are below the current detection limit.

The authors thank Claudio Pellegrini, Jan-Eric Rubinsson, and Sébastien Boutet for valuable discussions and comments, and Matt Hayes and the CXI technical staff for experimental support. Use of the Linac Coherent Light Source (LCLS), SLAC National Accelerator Laboratory, is supported by the U.S. Department of Energy, Office of Science, Office of Basic Energy Sciences under Contract No. DE-AC02-76SF00515. Use of the Stanford Synchrotron Radiation Lightsource, SLAC National Accelerator Laboratory, is supported by the U.S. Department of Energy, Office of Science, Office of Basic Energy Sciences under Contract No. DE-AC02-76SF00515. The
SSRL Structural Molecular Biology Program (T. K.) is supported by the DOE Office of Biological and Environmental Research, and by the National Institutes of Health, National Institute of General Medical Sciences (including P41GM103393). The contents of this publication are solely the responsibility of the authors and do not necessarily represent the official views of NIGMS or NIH. A part of this work was supported by the Director, Office of Science, Office of Basic Energy Sciences (OBES), Division of Chemical Sciences, Geosciences, and Biosciences (CSGB) of the Department of Energy (DOE) (Contract No. DE-AC02-05CH11231, J. Y., J. K., V. K. Y.) for x-ray methodology and instrumentation, and spectroscopy data collection and analysis, National Institutes of Health (NIH) Grants No. GM055302 (V. K. Y.), No. GM110501 (J. Y.), No. GM126289 (J. K.) for instrumentation development for $\mathrm{X}$-ray free-electron laser experiments, the Ruth L. Kirschstein National Research Service Award (F32GM116423, F. D. F.), and the Human Frontiers Science Project Award No. RGP0063/2013 310 (J. Y., U. B.) and the Department of Energy, Laboratory Directed Research and Development program at SLAC National Accelerator Laboratory, under Contract No. DE-AC02-76SF00515 (U. B., Y.Z.). F. D. F acknowledges the W. K. H. Panofsky Fellowship at SLAC National Accelerator Laboratory.

*tkroll@slac.stanford.edu

†clemens.weninger@maxiv.lu.se

*nina.rohringer@mpsd.mpg.de

§bergmann@slac.stanford.edu

[1] F. M. F. de Groot, A. Fontainet, C. C. Kao, and M. Krisch, Charge transfer multiplet calculations of the $\mathrm{K}$ beta X-ray emission spectra of divalent nickel compounds, J. Phys. Condens. Matter 6, 6875 (1994).

[2] U. Bergmann, M. M. Grush, C. R. Horne, P. De Marois, J. E. Penner-Hahn, C. F. Yocum, D. W. Wright, C. E. Dube, W. H. Armstrong, G. Christou, H. J. Eppley, and S. P. Cramer, Characterization of the Mn oxidation states in photosystem II by $\mathrm{K} \beta$ x-ray fluorescence spectroscopy, J. Phys. Chem. B 102, 8350 (1998).

[3] J. Badro, G. Fiquet, F. Guyot, J. P. Rueff, V. V. Struzhkin, G. Vanko, and G. Monaco, Iron partitioning in Earth's mantle: Toward a deep lower mantle discontinuity, Science 300, 789 (2003).

[4] P. Glatzel and U. Bergmann, High resolution 1s core hole Xray spectroscopy in $3 \mathrm{~d}$ transition metal complexes-electronic and structural information, Coord. Chem. Rev. 249, 65 (2005).

[5] G. Vanko, T. Neisius, G. Molnar, F. Renz, S. Karpati, A. Shukla, and F. M. F. de Groot, Probing the $3 \mathrm{~d}$ spin momentum with X-ray emission spectroscopy: The case of molecular-spin transitions, J. Phys. Chem. B 110, 11647 (2006).

[6] W. Zhang et al., Tracking excited-state charge and spin dynamics in iron coordination complexes, Nature (London) 509, 345 (2014). 
[7] M. W. Mara, R. G. Hadt, M. E. Reinhard, T. Kroll, H. Lim, R. W. Hartsock, R. Alonso-Mori, M. Chollet, J. M. Glownia, S. Nelson, D. Sokaras, K. Kunnus, K. O. Hodgson, B. Hedman, U. Bergmann, K. J. Gaffney, and E. I. Solomon, Metalloprotein entatic control of ligand-metal bonds quantified by ultrafast x-ray spectroscopy, Science 356, 1276 (2017).

[8] H. Visser, E. Anxolabhre-Mallart, U. Bergmann, P. Glatzel, J. Robblee, S. P. Cramer, J. J. Girerd, K. Sauer, M. P. Klein, and V. K. Yachandra, Mn K-Edge XANES and K $\beta$ XES studies of two Mn-Oxo binuclear complexes: Investigation of three different oxidation states relevant to the oxygenevolving complex of photosystem II, J. Am. Chem. Soc. 123, 7031 (2001).

[9] J. Kern et al., Simultaneous femtosecond X-ray spectroscopy and diffraction of photosystem II at room temperature, Science 340, 491 (2013).

[10] K. Haldrup et al., Observing solvation dynamics with simultaneous femtosecond X-ray emission spectroscopy and X-ray scattering, J. Phys. Chem. B 120, 1158 (2016).

[11] N. Rohringer, D. Ryan, R. A. London, M. Purvis, F. Albert, J. Dunn, J. D. Bozek, C. Bostedt, A. Graf, R. Hill, S. P. HauRiege, and J. J. Rocca, Atomic inner-shell X-ray laser at 1.46 nanometres pumped by an X-ray free-electron laser, Nature (London) 481, 488 (2012).

[12] M. Beye, S. Schreck, F. Sorgenfrei, C. Trabant, N. Pontius, C. Schüßler-Langeheine, W. Wurth, and A. Föhlisch, Stimulated X-ray emission for materials science, Nature (London) 501, 191 (2013).

[13] H. Yoneda, Y. Inubushi, K. Nagamine, Y. Michine, H. Ohashi, H. Yumoto, K. Yamauchi, H. Mimura, H. Kitamura, T. Katayama, T. Ishikawa, and M. Yabashi, Atomic innershell laser at 1.5-ångström wavelength pumped by an X-ray free-electron laser, Nature (London) 524, 446 (2015).

[14] T. Kroll, C. Weninger, R. Alonso-Mori, D. Sokaras, D. Zhu, L. Mercadier, V. P. Majety, A. Marinelli, A. Lutman, M. W. Guetg, F.-J. Decker, S. Boutet, A. Aquila, J. Koglin, J. Koralek, D. P. DePonte, J. Kern, F. D. Fuller, E. Pastor, T. Fransson, Y. Zhang, J. Yano, V. K. Yachandra, N. Rohringer, and U. Bergmann, Stimulated X-Ray Emission Spectroscopy in Transition Metal Complexes, Phys. Rev. Lett. 120, 133203 (2018).

[15] C. Weninger, M. Purvis, D. Ryan, R. A. London, J. D. Bozek, C. Bostedt, A. Graf, G. Brown, J. J. Rocca, and N. Rohringer, Stimulated Electronic X-Ray Raman Scattering, Phys. Rev. Lett. 111, 233902 (2013).

[16] T. E. Glover, D. M. Fritz, M. Cammarata, T. K. Allison, S. Coh, J. M. Feldkamp, H. Lemke, D. Zhu, Y. Feng, R. N. Coffee, M. Fuchs, S. Ghimire, J. Chen, S. Shwartz, D. A. Reis, S. E. Harris, and J. B. Hastings, X-ray and optical wave mixing, Nature (London) 488, 603 (2012).

[17] M. Fuchs et al., Anomalous nonlinear X-ray Compton scattering, Nat. Phys. 11, 964 (2015).

[18] S. Schulz, I. Grguraš, C. Behrens, H. Bromberger, J. T. Costello, M. K. Czwalinna, M. Felber, M. C. Hoffmann, M. Ilchen, H. Y. Liu, T. Mazza, M. Meyer, S. Pfeiffer, P. Prędki, S. Schefer, C. Schmidt, U. Wegner, H. Schlarb, and A. L. Cavalieri, Femtosecond all-optical synchronization of an X-ray free-electron laser, Nat. Commun. 6, 5938 (2015).
[19] P. Emma et al., First lasing and operation of an ångstromwavelength free-electron laser, Nat. Photonics 4, 641 (2010).

[20] T. Ishikawa et al., A compact X-ray free-electron laser emitting in the sub-ångström region, Nat. Photonics 6, 540 (2012).

[21] H. Kang et al., Hard X-ray free-electron laser with femtosecond-scale timing jitter, Nat. Photonics 11, 708 (2017).

[22] E. Allaria et al., Highly coherent and stable pulses from the FERMI seeded free-electron laser in the extreme ultraviolet, Nat. Photonics 6, 699 (2012).

[23] H. Weise and W. Decking, Commissioning and first lasing of the european XFEL, in Proceedings of the 38th International Free-Electron Laser Conference (FEL2017) (JACoW, Geneva, 2017), pp. 9-13, https://doi.org/10 .18429/JACoW-FEL2017-MOC03.

[24] C. Milne et al., SwissFEL: The swiss X-ray free electron laser, Appl. Sci. 7, 720 (2017).

[25] A. Benediktovitch, V. P. Majety, and N. Rohringer, Quantum theory of superfluorescence based on two-point correlation functions, Phys. Rev. A 99, 013839 (2019).

[26] L. Mercadier, A. Benediktovitch, C. Weninger, M. A. Blessenohl, S. Bernitt, H. Bekker, S. Dobrodey, A. Sanchez-Gonzalez, B. Erk, C. Bomme, R. Boll, Z. Yin, V. P. Majety, R. Steinbrügge, M. A. Khalal, F. Penent, J. Palaudoux, P. Lablanquie, A. Rudenko, D. Rolles, J. R. Crespo López-Urrutia, and N. Rohringer, Evidence of Extreme Ultraviolet Superfluorescence in Xenon, Phys. Rev. Lett. 123, 023201 (2019).

[27] M. Liang, G. J. Williams, M. Messerschmidt, M. M. Seibert, P. A. Montanez, M. Hayes, D. Milathianaki, A. Aquila, M. S. Hunter, J. E. Koglin, D. W. Schafer, S. Guillet, A. Busse, R. Bergan, W. Olson, K. Fox, N. Stewart, R. Curtis, A. A. Miahnahri, and S. Boutet, The coherent X-ray imaging instrument at the Linac Coherent Light Source, J. Synchrotron Radiat. 22, 514 (2015).

[28] A. A. Lutman, R. Coffee, Y. Ding, Z. Huang, J. Krzywinski, T. Maxwell, M. Messerschmidt, and H.-D. Nuhn, Experimental Demonstration of Femtosecond Two-Color X-Ray Free-Electron Lasers, Phys. Rev. Lett. 110, 134801 (2013).

[29] M. W. Guetg, A. A. Lutman, Y. Ding, T. J. Maxwell, F.-J. Decker, U. Bergmann, and Z. Huang, Generation of HighPower High-Intensity Short X-Ray Free-Electron-Laser Pulses, Phys. Rev. Lett. 120, 014801 (2018).

[30] B. Nagler, A. Aquila, S. Boutet, E. C. Galtier, A. Hashim, M. S. Hunter, M. Liang, A. E. Sakdinawat, C. G. Schroer, A. Schropp, M. H. Seaberg, F. Seiboth, T. van Driel, Z. Xing, Y. Liu, and H. J. Lee, Focal Spot and Wavefront Sensing of an X-Ray Free Electron laser using Ronchi shearing interferometry, Sci. Rep. 7, 13698 (2017).

[31] M. H. Seaberg, A. Aquila, M. Liang, H. J. Lee, B. Nagler, Y. Liu, A. Sakdinawat, F. Seiboth, M. Makita, Y. Sun, R. Signorato, S. Carbajo, Y. Feng, J. Krzywinski, D. Zhu, and S. Boutet, Nanofocus characterization at the Coherent X-ray Imaging instrument using 2D single grating interferometry, SPIE 11038, 110380L (2019).

[32] S. Herrmann, S. Boutet, B. Duda, D. Fritz, G. Haller, P. Hart, R. Herbst, C. Kenney, H. Lemke, M. Messerschmidt, J. Pines, A. Robert, M. Sikorski, and G. Williams, CSPAD-140k: 
A versatile detector for LCLS experiments, Nucl. Instrum. Methods Phys. Res., Sect. A 718, 550 (2013).

[33] R. Alonso-Mori et al., Energy-dispersive X-ray emission spectroscopy using an X-ray free-electron laser in a shot-byshot mode, Proc. Natl. Acad. Sci. U.S.A. 109, 19103 (2012).

[34] G. Hölzer, M. Fritsch, M. Deutsch, J. Härtwig, and E. Förster, $K \alpha_{1,2}$ and $K \beta_{1,3}$ x-ray emission lines of the $3 d$ transition metals, Phys. Rev. A 56, 4554 (1997).

[35] C. Weninger and N. Rohringer, Transient-gain photoionization x-ray laser, Phys. Rev. A 90, 063828 (2014).

[36] D. Attwood and A. Sakdinawat, X-Rays and Extreme Ultraviolet Radiation, Principles and Applications (Cambridge University Press, Cambridge, England, 2017), ISBN-10: 1107062896, ISBN-13: 978-1107062894.
[37] C. Pellegrini, A. Marinelli, and S. Reiche, The physics of x-ray free-electron lasers, Rev. Mod. Phys. 88, 015006 (2016).

[38] V. Kimberg and N. Rohringer, Stochastic stimulated electronic x-ray Raman spectroscopy, Struct. Dyn. 3, 034101 (2016).

[39] Y. Liu, M. Seaberg, D. Zhu, J. Krzywinski, F. Seiboth, C. Hardin, D. Cocco, A. Aquila, B. Nagler, H. J. Lee, and S. Boutet, High-accuracy wavefront sensing for $\mathrm{X}$-ray free electron lasers, Optica 5, 967 (2018).

[40] D. Zhu, M. Cammarata, J. M. Feldkamp, D. M. Fritz, J. B. Hastings, S. Lee, H. T. Lemke, A. Robert, J. L. Turner, and Y. Feng, A single-shot transmissive spectrometer for hard X-ray free electron lasers, Appl. Phys. Lett. 101, 034103 (2012). 